\title{
Humanoid Based Intelligence Control Strategy of Plastic Cement Die Press Work-Piece Forming Process for Polymer Plastics
}

\author{
Dekuan Liu', Shuang Jin², Hu Xu \\ ${ }^{1}$ International College, Chongqing Jiaotong University, Chongqing, China \\ ${ }^{2}$ College of Automation, Chongqing University, Chongqing, China \\ Email: 1751332285@qq.com,884580930@qq.com
}

Received 29 April 2016; accepted 20 May 2016; published 24 May 2016

\begin{abstract}
The plastic cement belongs to a sort of polymer material, the chemical composition is very complex, and the plastic cement work-piece is generally manufactured by die press forming. Aimed at being difficult to control in parameters of forming process, the paper explored the humanoid based intelligence control strategy. In the paper, it made the anatomy in control puzzle resulted in uncertainty such as chemical component of plastic cement etc., summarized up the characteristic of cybernetics in forming process, researched on the humanoid based intelligence control strategy, and constructed the control algorithm of forming process in plastic cement work-piece. Taking the process experiment of temperature and pressure control as an example, it validated the good dynamic and static control quality through simulation of control algorithm constructed in this paper. The experimental results show that the control algorithm explored in this paper is reasonably available.
\end{abstract}

\section{Keywords}

Plastic Cement, Polymer Plastics, Die Press Work-Piece, Forming Process, Humanoid Based Intelligence Control

\section{Introduction}

The plastic work-piece owns a wide application in engineering field, however, the physical and chemical properties of the products are restricted by many factors such as compound formula of sizing material, structure size, molding process, processing environment and so on. Due to the complex technics in the production process, the control of the production process mainly depends on experience and skills of the operator at present, and the stability of product quality is controlled by the subjective initiative of the operator in a great extent. In view of the current production process control is difficult to guarantee the stability of product quality and to avoid being 
influenced by human factors, the key improved the stability of product quality is to adopt the correct control strategy, and therefore, this paper explores the control strategy of the plastic work-piece forming process.

\section{Control Puzzle of Forming Process}

Under the specified conditions, the forming control is the physical and chemical process which makes the polymer plastics compound material and various additives produce the cross-linking chemical reaction. Except the control process is influenced by the factors such as product formula etc, the key factor which has a decisive impact on the performance of the product is to control the forming temperature, temperature holding time and injection pressure of forming process. If a certain pressure is applies to the die press work-piece forming process, then it can promote the flow of plastic material in the mold cavity, and eliminate air bubbles so as to ensure the compactness of the product. The pressure depends on the product structure, the nature of the material and the technics conditions. For example, if it is poor in mobility of plastic material, in order to eliminate the bubble and make the plastic material be able to be filled in the whole mold cavity quickly, and then the pressure should be larger. Due to the plastic being a polymer material, even if it is the same material, its difference of the molecular weight can probably be tens of thousands of times, so the forming process is full of serious uncertainty. In the forming process of plastic product, the temperature field distribution in the mold cavity is not uniform, the heat transfer is obviously lagging, it belongs to the non-steady state system, and therefore, there is obvious the strong nonlinearity. The thermodynamic property is varied with chemical reaction because of the change of heat transfer and heat capacity of plastic material, and so the heat transfer lagging is unknown and time variant. The plastic material is a bad heat conductor, the thermal conductivity is related to the temperature and so on, and so its thermodynamic parameter has a great uncertainty. In the forming technics of molded plastic products, the technical key that influences the stability of product forming quality is to actualize the optimization control for injection pressure, process temperature and forming time of the forming process. The above is just the control puzzle currently faced on the forming process control, and as to what kind of control strategy should be taken, it deals with the study on the cybernetics characteristic of the forming process. The following explores the related control strategy.

\section{Cybernetics Characteristic and Control Strategy of Forming Process}

Process cybernetics characteristics. Based on the analysis of control puzzle in work-piece forming process mentioned above, the cybernetics characteristics of forming process can be summarized up as the following [1] [2].

1) Uncertainty of process parameters. Because there is any fixed chemical formula of the plastics material, the forming process is closely related to the factors such as the nature of the plastics material formula and so on, and a variety of uncertainties lead to the randomness of the process parameters, distribution, time varying and the unknown. It is to say that the process parameter given in the design is only a reference value of estimation.

2) Large inertia and pure lag characteristics of forming process. The plastic material is not a good conductor of heat transfer, and there is obvious the heat transfer inertia and lag characteristics.

3) Strong nonlinear characteristics. The thermodynamic equation itself is a nonlinear differential equation or partial differential equation, and so the whole molding process is strongly nonlinear.

4) Time variant and unknown of processes time lag. In the chemical reaction process, the time variability and the unknown of the time lag are bound to be caused by uncertainty because of its material properties are constantly changing.

5) In the process of forming process, the environmental disturbances are not predictable and own the diversity. In the face of the above cybernetics characteristics, it is impossible to obtain the expected control effect with the conventional control strategy, and therefore, it is necessary to seek new control strategy.

Control strategy selection. The forming process is too difficult to be mathematically modeled, and the conventional paradigm control strategy is not desirable because of it is impossible to achieve optimal control. It can only provide the selection is the intelligent control strategy. Such as fuzzy control strategy has currently been a lot of successful application cases, but in the face of uncertainty forming process control, the control effect is not ideal, and it is obviously powerless because of the membership function can not be determined. The expert control system can handle various kinds of the information including the precise, fuzzy, qualitative and quantitative information, but in the forming process of uncertainty, the acquisition of relevant experienced knowledge is not 
so easy, and it is very difficult to provide control mode for forming process control. The HSIC (Human Simulated Intelligent Controller) or humanoid based intelligent controller is the isomorphic transformation of human control system, and it simulates the human behavior and is particularly worthy of attention. The HSIC control strategy is different from the PID conventional control, and it adopts generalized knowledge feature model to implement the control for controlled process. Both the dynamic and static characteristics of the process are considered, it researches on the control strategy, control mode and control algorithm of the process from the concept of system hierarchy, and therefore, it has the notable characteristics such as being high in control precision, short in transient process time, strong in robustness, no overshoot, and easy in integrating the experience, skill and wisdom etc of the actual field operator. The advantage of humanoid based intelligent control strategy is to imitate the control behavior of the control expert, and it can implement the multi-mode control of humanoid based intelligence for forming process based on the integration of a variety of knowledge. As the control strategy is to take into account for the need to dynamic and static quality index of the process in rapidity and smoothness, robustness and accuracy and so on, it is more close to the actual engineering application. The successful engineering practice has demonstrated the superiority of the explored control strategy [3]-[5], and therefore, the selects the strong robustness control strategy of humanoid based intelligence in this paper.

\section{Control Model and Control Algorithm}

Control model structure. In order to research the control algorithm, it is necessary to explore the control model and controller characteristic. The generalized control model of humanoid based intelligence is essentially an intelligent program system. It firstly makes the integration of the user index and the knowledge base and fuses the integrated result into a generalized knowledge set, and then the knowledge set, the inference mechanism and the control rules are integrated into a humanoid based intelligent controller. The model structure of the generalized control model is shown as in Figure 1, and in Figure 1, the $r(t), e(t), u(t), y(t)$ is the process input, process error, controller output and controlled process output. For convenience in the following discussion, the $e(t)$ is shown by $e$, and its change rate is shown by $\dot{e}$.

Control algorithm. Analyzing the relationship between the process error $e$ and the change rate $\dot{e}$ of the process error, the error phase plane can be formed as shown in Figure 2, and from control theory, it can summarize out the error feature mode of the control process. When the process error $e$ lies in the second and the fourth quadrant of the error phase plane, namely $e \cdot \dot{e}<0$ or $\dot{e}=0$, the process error would represent the reduced trend and gradually approach to zero. When the process error $e$ lies in the first and third quadrant of the error phase plane, namely $e \cdot \dot{e}>0$ or $e=0$ and $\dot{e} \neq 0$, the process error shows an increasing trend. Aimed at the different two kinds of error feature mode, its corresponding control strategy is that when $e \cdot \dot{e}<0$ or $\dot{e}=0$, because it makes automatically the process error gradually approach to reducing, and therefore, the "hold" control mode can be selected. The advantage of this control mode is that it can eliminate the integral saturation and the phase lag problem without increasing the integral node. When $e \cdot \dot{e}>0$ or $e=0$ and $\dot{e} \neq 0$, because the negative feedback proportional control can reduce the error, therefore, the proportional control mode can be selected.

In order to achieve fine control, the error phase plane can be divided into symmetrical and different control regions according to the process error $e$ and the change rate $\dot{e}$ of the process error being different, and the error phase plane shown as in Figure 2 can be divided into different control areas. For example, it can be set in advance to the two threshold, such as $A b s\left(e_{1}\right)>A b s\left(e_{2}\right)$, and $\dot{e}$ set a threshold $A b s\left(\dot{e}_{1}\right)$, then the whole error phase plane can be divided into 6 control areas. Aimed at the different error feature model of different regions, it can take different control strategy, and so the control algorithm of the different error feature is constructed.

1) If $A b s(e) \geq A b s\left(e_{1}\right)$, regardless the change rate of process error is how to take the value, aiming at the characteristics of the process error being large, and the process error must be reduced. Therefore, the Bang-Bang control strategy is the best selection because of it can most rapidly reduce the error of the process.

2) If $e \cdot \dot{e}<0$ but $A b s(\dot{e}) \ngtr A b s\left(\dot{e}_{1}\right)$, then the proportional control mode is desirable because of the



Figure 1. Generalized control model. 


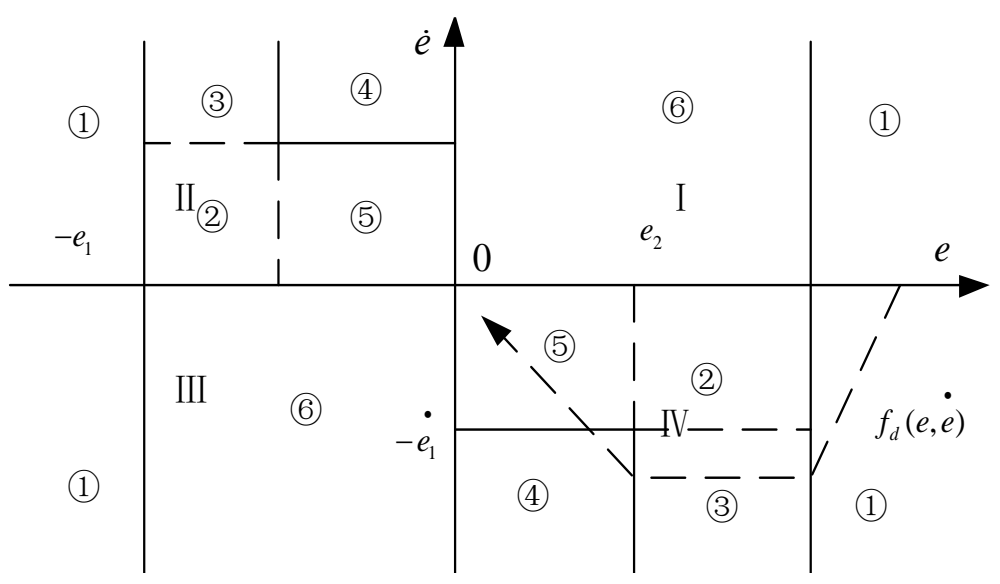

Figure 2. Error phase plane.

process error being reducing. Since the joining of differential control can accelerate the change of the error rate, it can adopt the proportional plus derivative control strategy.

3) If $e \cdot \dot{e}>0, A b s(e)<A b s\left(e_{1}\right)$, the value of $A b s(\dot{e})$ can be taken in arbitrary value, due to the joining of differential control can accelerate the change of error change rate, and so the proportional plus derivative control strategy is desirable.

4) If $e \cdot \dot{e}<0$, but $A b s(\dot{e})>A b s\left(\dot{e}_{1}\right)$, then in order to accelerate the change of error change rate, the proportional feedback differential control strategy is available.

5) If $e \cdot \dot{e}<0, A b s(\mathrm{e})<A b s\left(e_{2}\right), A b s(\dot{e})<A b s\left(\dot{e}_{1}\right)$, in the case of both process error and its change rate all reach the expected value, then in order to eliminate the steady state error of process control, the proportional plus integral control strategies are available.

Summarizing up the above five pieces of control rule, the control algorithm can be expressed as the following.

$$
u_{n}= \begin{cases}\operatorname{sgn}\left(e_{n}\right) U_{\max } & \left(A b s(\mathrm{e}) \geq A b s\left(\mathrm{e}_{1}\right)\right) \\ K_{p} \cdot e_{n} & \left(A b s(\dot{e}) \geq A b s\left(\dot{e}_{1}\right)\right) \\ K_{p} \cdot e_{n}+K_{d} \cdot \dot{e}_{\mathrm{n}} & \left(A b s(\dot{e}) \geq A b s\left(\dot{e}_{1}\right), A b s(\mathrm{e}) \geq A b s\left(\mathrm{e}_{2}\right)\right) \\ -K_{p}^{\prime} \cdot e_{n}+K_{d}^{\prime} \cdot \dot{e}_{\mathrm{n}} & \left(A b s(\dot{e}) \geq A b s\left(\dot{e}_{1}\right), A b s(\mathrm{e}) \leq A b s\left(\mathrm{e}_{2}\right)\right) \\ -K_{p} \cdot e_{n}+K_{i} \cdot \sum_{i=1}^{n} e_{m, i} & \left(A b s(\dot{e}) \leq A b s\left(\dot{e}_{1}\right), A b s(\mathrm{e}) \leq A b s\left(\mathrm{e}_{2}\right)\right)\end{cases}
$$

In the control algorithm, $e_{n}$ is the $n$th process error, $e_{m, j}$ is the $i$ th extremum of process error, $\dot{e}_{n}$ is the $n$th change rate of process error, $u_{n}$ is the $n$th output of controller, $U_{\max }$ is the Maximum of controller output, $K_{d}, K_{d}^{\prime}$ is respectively the differential coefficient, $K_{p}, K_{p}^{\prime}$ is respectively the proportional coefficient, and the $K_{i}$ is the integral coefficient.

Essentially the control algorithm is to simulate the human thinking process, and according to the error feature model, it can construct various kinds of algorithm to make the control for the forming process [6] [7]. Based on combination with open loop control and closed loop control, there is only two sorts of basic process error dynamic feature mode [8]. Its prototype control algorithm can be summarized as follows:

$$
U=\left\{\begin{array}{cc}
K_{p} \cdot e+k \cdot K_{p} \cdot \sum_{i=1}^{n-1} e_{m, i} & (e \cdot \dot{e}>0 \text { or } e=0 \dot{e} \neq 0) \\
k \cdot K_{p} \cdot \sum_{i=1}^{n} e_{m, i} & (e \cdot \dot{e}<0 \text { or } \dot{e}=0)
\end{array}\right.
$$

In the prototype control algorithm, $k$ is the suppression coefficient, $U$ is the output of controller, and the other symbol meaning is the same as the previous mentioned above.

The above does not deal with the process performance specifications, such as the speed of response to the rise 
time, the length of time to adjust and so on, and therefore, it is necessary to integrate the advantages of other control strategies to meet the requirements of the process performance index for more complex process. For example, when the process error is very large, if it adds into the Bang-Bang control, then it can shorten the time of the rise and so on. In order to simplify the system control structure, it can make the knowledge of expert knowledge and the skill of the operator of the field such as control rules and knowledge base, etc. integrate into a body, and constitute a fusion controller based on humanoid intelligence, and adopt production control rule to realize the process control. The advantage of this strategy is that it is good for modularity and nature because there is no direct relationship between the control rules. According to different conditions, each rule can be independently increased, deleted and modified, and it has better environmental adaptability so as to constitute a strong robustness controller [9] [10].

\section{Simulation Experiment and Its Analysis}

Simulation experiment. In order to verify the strong robustness control performance of algorithm, the response of the process is studied from two aspects of strong external interference and process parameter change in the simulation experiment. Taking PID control as a reference, it makes the comparison of process response robustness, and observes the technical specification changes in rise time, adjusting time, whether overshoot, and steady state control precision and so on. Here it takes model as $W(s)=K e^{-\tau s} /(T s+1)$ to make the simulation. In which, $K$ is a gain coefficient, $T$ is the time constant of controlled object, $\tau$ is pure lag time of the system. For convenience, it takes $K=1, T=1.2, \tau=2$, then it can get $W(s)=e^{-2 s} /(1.2 s+1)$, Based on the environment of MATLAB, under the condition of unit step input, it makes the simulation for the same controlled object respectively by PID and HSIC controller. The response curve of simulation is shown as Figure 3.

In Figure 3, curve 1 and curve 2 are respectively the response curve of PID and HSIC controller. It can be seen that both curve 1 and curve 2 do not appear overshoot. But the rising and adjusting time of the former is longer than HSIC controller, and therefore it owns better control quality than PID controller.

The following makes comparison of simulation curves under the different conditions so as to explain HSIC control to be better than PID control. Figure 4 shows the curve which joins a pulse interference at $t=4.5 \mathrm{~s}$, it can be seen that HSIC controller has better anti-interference performance than PID control. Figure 5 shows the curve comparison which the open-loop gain $K$ changes only from $K=1$ to $K=2$, it can be seen that the overshoot of PID controller is enlarged much more, but it is still kept non-overshoot for HSIC controller.

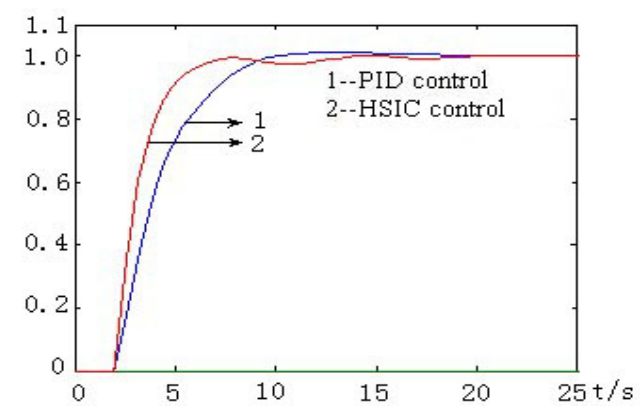

Figure 3. Process response curve.

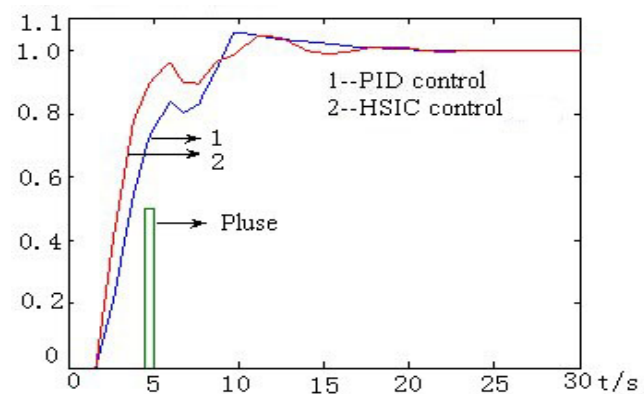

Figure 4. Response with a interference. 


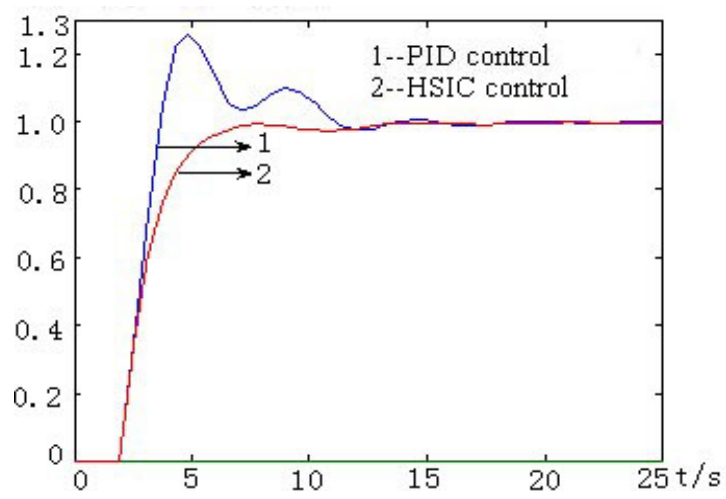

Figure 5. Response after gain $\mathrm{K}$ changed.

Figure 6 shows the response curve when $\tau$ is changed from $2 \mathrm{~s}$ to $4 \mathrm{~s}$, and it shows that the overshoot is more enlarged for PID controller, but for the curve of HSIC controller, in spite of there being a little change it is still hardly any change, and it is only to postpone $2 \mathrm{~s}$ in response time. Figure 7 shows the response curve when $\mathrm{T}$ changes from $1.2 \mathrm{~s}$ to $2 \mathrm{~s}$, and it is slightly a overshoot for PID controller, but there is hardly any change for HSIC controller in response curve.

Here it is worth to be mentioned that if it follows a inertia unit after controlled object, then the transfer function will be $W(S)=K e^{-\tau s} /(T s+1)(2 s+1)$, the result of simulation is shown as in Figure 8. From Figure 8, it can be seen that the overshoot enlarged for PID control, but for HSIC controller, there is hardly any changed in response curve, and special there is not any overshoot.

The above simulation shows that HSIC controller has better robustness and anti-interference performance than PID controller.

Experimental verification. Now taking a plastic bearing work-piece forming control as an example, the two control methods of PID and HSIC are respectively adopted to control the forming process in control system before and after reform, the expected pressure is set to $16 \mathrm{MPa}$, the range of the original process pressure change is from 16.50 to $15.12 \mathrm{MPa}$, and the change range of the process pressure is drom 16.14 to $15.84 \mathrm{MPa}$ through reform. In order to compare the control effect, the sampling period takes $100 \mathrm{mS}$, the continuous sampling time is 10 minutes, the pressure average value, variance and range of two kinds of pressure control system can be obtained according to the data processing of sample record, and the statistical values of the process pressure parameters are shown as in Table 1. Compared with the data in Table 1, it can be known that HSIC control effect is obviously better than the control effect of PID. In the same way, the statistical values of the process temperature parameters of the PID and HSIC two control methods are compared, if the temperature value is set to $165^{\circ} \mathrm{C}$ before and after the system reform, then the observation record shows that the temperature range of the present process is from $163.0^{\circ} \mathrm{C}$ to $166.5^{\circ} \mathrm{C}$ after reform, and the temperature range of the original process is from $152.0^{\circ} \mathrm{C}$ to $173.0^{\circ} \mathrm{C}$. The sampling period and sampling time are the same as those of the above pressure system, and the average value of the system temperature parameters, the variance, the range of statistical values are shown as in Table 2.

Analysis of simulation and experiment. The simulation of the process shows that the HSIC control has the strong robustness for the process control of forming process in complex plastic work-piece. In addition, the engineering experimental data in Table 1 and Table 2 show that the control effect of HSIC control algorithm is more ideal, owns a relatively strong engineering adaptability in aspects of control accuracy, mean, variance, and range and so on.

\section{Conclusions}

The key to control the process of plastic molding is to control the process temperature, injection pressure and holding time. In view of the large inertia and large time lag characteristic of the controlled process, it suffers from lots of influence in multiple factors with uncertainty, and the control of forming process is very difficult. Simulation experiment and engineering practice of HSIC strategy proposed in this paper show that the control strategy is feasible and effective, and it provides a useful reference for work-piece forming process control in 


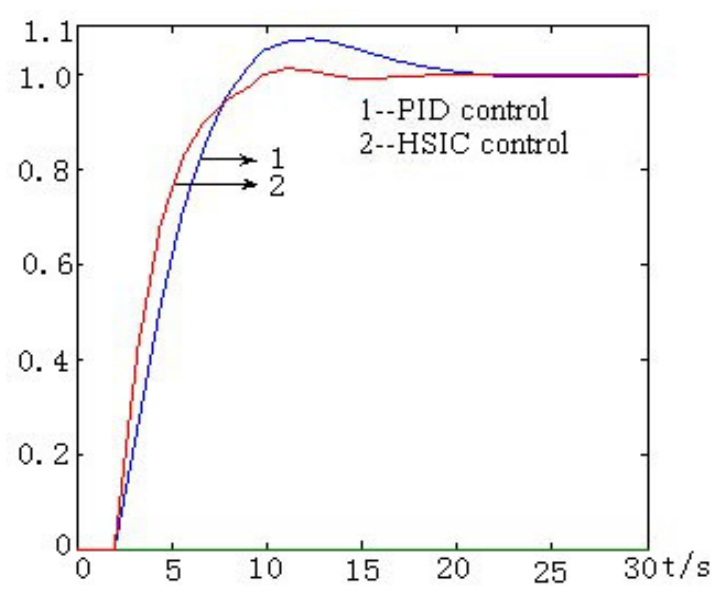

Figure 6. Response with time-lag changed.

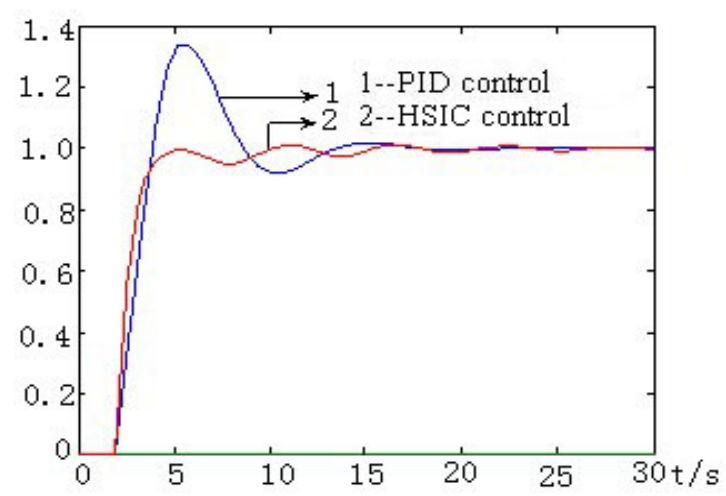

Figure 7. Response after $\mathrm{T}$ changed.

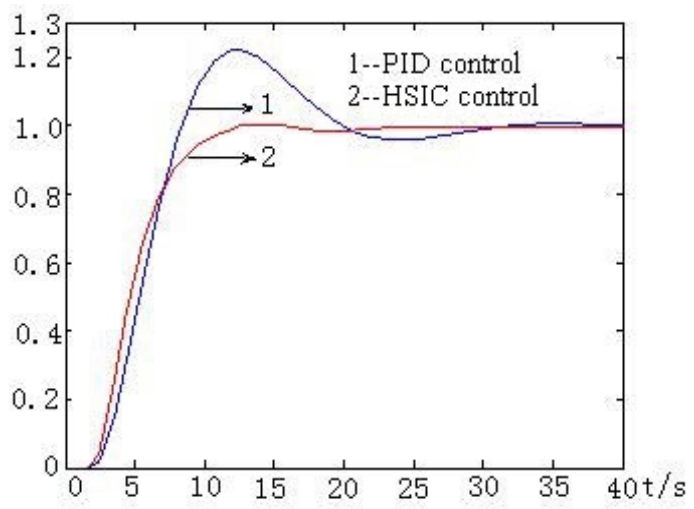

Figure 8. Response changing as a 2-order system.

Table 1. Comparison of statistical values of process pressure parameters.

\begin{tabular}{|c|c|c|c|}
\hline System Statistical value & Average value & Variance & Range \\
\hline Original system & 15.82 & 0.62 & 1.38 \\
\hline Current system & 15.97 & 0.12 & 0.30 \\
\hline
\end{tabular}


Table 2. Comparison of statistical values of process temperature parameters.

\begin{tabular}{cccc}
\hline \multicolumn{1}{c}{ System } & Average value & Variance & Range \\
\hline Original system & 163.2 & 8.0 & 21.0 \\
Current system & 164.7 & 1.1 & 3.5 \\
\hline
\end{tabular}

polymer plastics.

\section{References}

[1] Peng, L., Lin, Y. and Yang, Y. (2004) Exploring on Related Technology in Complex System Control. Journal of Southwestern Normal University (Natural Science Edition), 29, 1066-1068.

[2] Pei, Y.L. (2013) Intelligent Fusion Control Strategy for Complex Vulcanization Process. Machine Tools and Hydraulic, 41, 92-96.

[3] Xiong, X.S. (2013) Strong Robustness Control Strategy of Complex Combustion Process with Uncertainty. Journal of Chongqing University of Technology (Natural Science Edition), 27, 106-110.

[4] Wang, J. and Xiao, C. (2013) Multi-Modal Human Simulated Intelligent Control Strategy for Complex Process. Microelectronics, 43, 221-224.

[5] Wang, J. (2012) Intelligent Fusion Control Strategy for Aeration Process of Wastewater Treatment. Journal of Southwestern Normal University (Natural Science Edition), 34, 120-124.

[6] Li, S.Y. (2002) Fuzzy Control • Neuron Control and Intelligent Cybernetics. Harbin Institute of Technology Press, Harbin.

[7] Yi, J.K. (2004) Intelligent Control Technology. Beijing University of Technology Press, Beijing.

[8] Li, Z.S. and Tu, Y.Q. (2003) Human Simulated Intelligent Controller. National Defense Industry Press, Beijing.

[9] Liu, Y.C., Liu, Y.B. and Li, T.F. (2008) Human Simulated Intelligent Control of the of Rubber Vulcanization Process. Microcomputer Information, 24, 80-82.

[10] Huang, H. and Bi, J.C. (2011) Application Research of Intelligent Fusion Control Strategy in Water Purification System. Journal of Southwestern Normal University (Natural Science Edition), 31, 162-166. 